\title{
Conductivity and Hall-effect in highly resistive GaN layers
}

\author{
P. Kordoša) and P. Javorka \\ Institute of Thin Film and Ion Technology, Research Centre Jülich, D-52425 Jülich, Germany \\ M. Morvic and J. Betko \\ Institute of Electrical Engineering, Slovak Academy of Sciences, SK-84239 Bratislava, \\ Slovak Republic
}

J. M. Van Hove, A. M. Wowchak, and P. P. Chow

SVT Associates, Eden Prairie, Minnesota 55344

(Received 28 March 2000; accepted for publication 26 April 2000)

\begin{abstract}
Highly resistive $\mathrm{GaN}$ layers grown by molecular beam epitaxy are characterized by temperature dependent conductivity and Hall effect measurements. Samples with $\rho_{300} \cong 3 \times 10^{3} \Omega \mathrm{cm}$ show room temperature Hall mobility of 22 and $35 \mathrm{~cm}^{2} \mathrm{~V}^{-1} \mathrm{~s}^{-1}$ and have a temperature dependence $\mu_{H} \sim T^{x}$ with $x=0.9$ and 0.5 . This is in contradiction to a sample with $\rho_{300} \cong 32 \Omega \mathrm{cm}$ which has a room temperature mobility of $310 \mathrm{~cm}^{2} \mathrm{~V}^{-1} \mathrm{~s}^{-1}$ and a $\mu_{H} \sim T^{x}$ with $x=-1.4$. The same activation energy of $0.23 \mathrm{eV}$, attributed to donor-like defects, is found for all three samples investigated. Temperature dependent conductivity data can be reasonably fitted considering band conduction. The presence of various hopping mechanisms is discussed. (C) 2000 American Institute of Physics.
\end{abstract}

[S0003-6951(00)02825-4]

Highly resistive GaN buffer layers are important for field-effect transistor and high electron mobility transistor structures to avoid a parallel conductive channel that degrades device performance. However, not much is reported about their preparation and transport properties. Layers with resistivity of $14,3 \times 10^{3}$, and $10^{6} \Omega \mathrm{cm}$ were previously prepared by varying the stoichiometry during molecular beam epitaxial (MBE) growth. ${ }^{1}$ Another possibility to obtain high resistive GaN layers is by acceptor doping. MBE grown $\mathrm{C}$-doped layers ${ }^{2}$ and hydride vapor phse epitaxial $\mathrm{Zn}$-doped layers ${ }^{3}$ with resistivity of $10^{6}$ and $10^{12} \Omega \mathrm{cm}$, respectively, have been reported. Unfortunately, carrier concentration and mobility data on samples with resistivity higher than 14 $\Omega \mathrm{cm}$ are not present in the literature due to the use of conductive substrates, ${ }^{3}$ or unmeasurable Hall coefficients. ${ }^{1,4}$ Hopping conduction, with extremely low Hall mobility $\mu_{H}$ $<1 \mathrm{~cm}^{2} \mathrm{~V}^{-1} \mathrm{~s}^{-1}$, is assumed to be responsible for the latter case. ${ }^{1,5}$ When the concentrations of the autodoping centers and the deep defects are comparable, the layer becomes highly resistive and conduction by hopping among deep centers might occur. Additional models taking into account scattering on charged dislocations resulting in reduced mobility have been proposed. ${ }^{6,7}$ To extend the knowledge of carrier transport behavior in $\mathrm{GaN}$ layers, we report in this letter on the temperature dependent conductivity and Hall effect measurements on highly resistive GaN layers grown by MBE.

The undoped GaN layers were grown on sapphire substrates by MBE using a radio frequency atomic nitrogen plasma source. $^{8}$ A low temperature AlN buffer was grown after nitridation of the sapphire. The substrate temperature was then increased to $750^{\circ} \mathrm{C}$ for the 2 - $\mu$ m-thick $\mathrm{GaN}$ growth which was done under slightly Ga-rich flux ratios. High resolution x-ray diffraction measurements showed a

${ }^{\text {a)} E l e c t r o n i c ~ m a i l: ~ p . k o r d o s @ f z-j u e l i c h . d e ~}$ reasonable layer quality with $\cong 7$ arc min full width at half maximum of the (0002) $\omega$ scan. ${ }^{9}$ Samples of about $6 \times 6 \mathrm{~mm}^{2}$ were cut from each wafer and In contacts were alloyed at $850{ }^{\circ} \mathrm{C}$ after appropriate surface cleaning. The ohmic behavior of the contacts was confirmed by current-voltage characteristics. Accurate temperature dependent conductivity and low magnetic field $(B=0.5 \mathrm{~T})$ Hall effect measurements were carried out using a high-impedance system.

The samples investigated can be characterized by the following room temperature resistivity and Hall mobility data: $\rho=3.6 \times 10^{3}, 2.6 \times 10^{3}$, and $32 \Omega \mathrm{cm}$ and $\mu_{H}=22,35$, and $310 \mathrm{~cm}^{2} \mathrm{~V}^{-1} \mathrm{~s}^{-1}$ for samples designated as $\mathrm{S} 1, \mathrm{~S} 2$, and S3, respectively. The temperature dependence of their conductivity and apparent Hall concentrations are shown in Fig. 1. The experimental data of both conductivity and concentration can be well fitted considering that thermally activated carriers dominate, $\sigma$ and $n_{H} \sim \exp \left(-E_{d} / k T\right)$. Note that the carriers are electrons deduced from the negative Hall coefficient. The activation energies evaluated from the $\sigma$ vs $1 / T$ dependence, commonly used in literature, are $0.30,0.28$, and $0.24 \mathrm{eV}$ for S1, S2, and S3 samples, respectively. However, the same activation energy $E_{d}=0.23 \mathrm{eV}$ is obtained for all three samples from fitting of our experimental data plotted as $n_{H} / T^{3 / 2}$ vs $1 / T$ (not shown here). All these values are evidently higher than the reported values of 0.016 and $0.11 \mathrm{eV}$ for similarly resistive MBE GaN. ${ }^{1}$ Recently the preparation of high resistive $n$-GaN layers by implantation with various ions has been reported and defect levels of $0.20-0.49 \mathrm{eV}$, evaluated from the $\rho$ vs $1 / T$ dependence, have been found. ${ }^{10}$ Our data are in good agreement with this result, what indicates that donor-like defects play a dominant role in the conduction mechanism in the samples investigated.

The temperature dependence of the apparent Hall mobility (in $\log -\log$ scale) for all three samples is shown in Fig. 2. The experimental data are fitted according to $\mu_{H} \sim T^{x}$ dependence. As expected from the room temperature data, differ- 


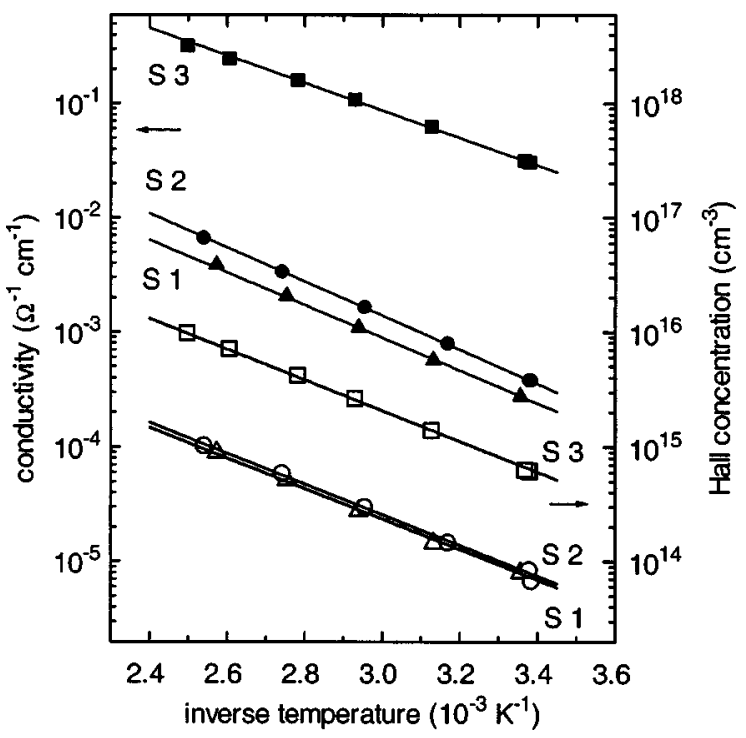

FIG. 1. Temperature dependence of conductivity and apparent Hall concentration for highly resistive GaN layers. The solid lines are theoretical fits. Note that full marks belong to the left axis and open marks to the right axis.

ent dependence of the Hall mobility on temperature is obtained for S3 compared with S1 and S2 samples. A scattering power $x=-1.4$ is found for S3 (note that $n_{H}=6$ $\times 10^{14} \mathrm{~cm}^{-3}$ at $300 \mathrm{~K}$ ), which indicates that phonon scattering dominates in conduction of this sample. On the other hand, for S1 and S2 samples the Hall mobility increases with increasing temperature and from fitting of the experimental data power values of $x=0.9$ and 0.5 are obtained. According to the dislocation scattering theory ${ }^{11}$ the drift mobility depends on temperature as $\mu_{\text {disl }}=C T^{3 / 2} / \lambda$, where $\lambda$ is the screening parameter which depends on temperature as $\sim T^{1 / 2}$ (neglecting temperature dependence of the effective screening concentration). Thus, for the scattering on charged dislocations a mobility vs temperature dependence $\mu_{\text {disl }}=C T$ should exist. From this it follows that conduction in samples S1 and S2 with the scattering power near unity should be

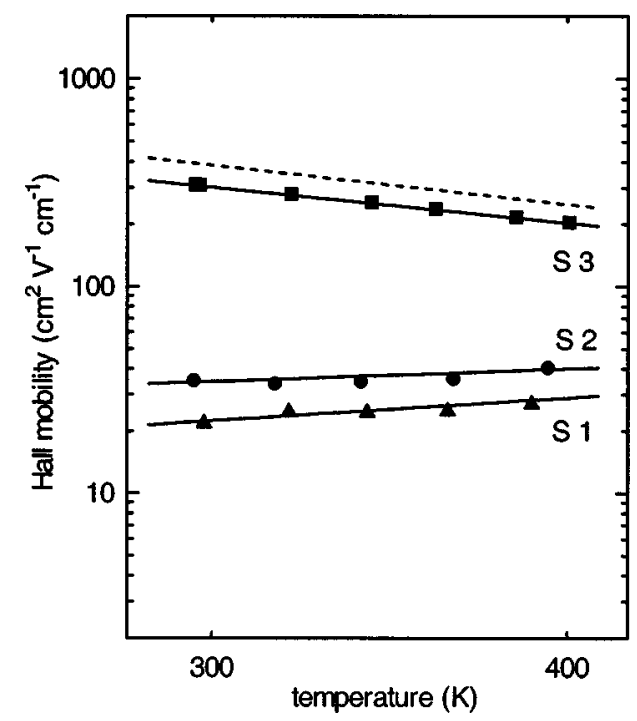

FIG. 2. Temperature dependence of Hall mobility (log-log scale) for analyzed GaN layers. The solid lines are theoretical fits for $\mu_{H} \sim T^{x}$ and the dashed line represents a dependence with $x=-1.5$. and $\epsilon_{3}$ values are again unrealistic. Thus, the question of
dashed line represents a dependence with $x=-1.5$.

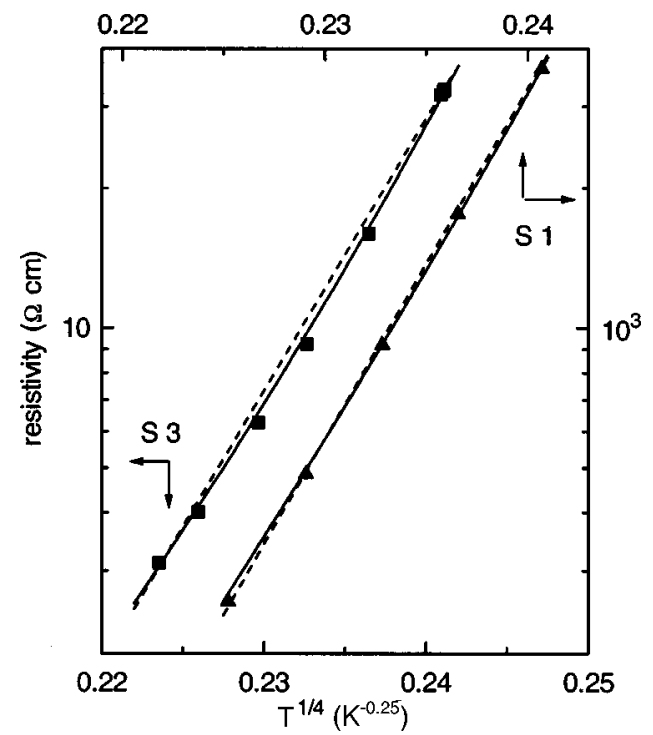

FIG. 3. Resistivity vs $T^{-1 / 4}$ dependence for two GaN layers. The solid and dashed lines are theoretical fits for multiphonon and variable range single phonon hopping, respectively.

mainly by interaction with dislocations, in contradiction to the sample S3.

Further, we will discuss the data obtained on samples investigated from the hopping conduction point of view. It has been reported that the conduction mechanism in GaN with a high number of defect centers does not consist of transport in the conduction band but of hopping among localized defects. ${ }^{1,5}$ It was found that the experimental data are most consistent with multiphonon hopping, rather than nearest-neighbor or variable range single phonon hopping. We have analyzed our data considering all three types of hopping.

For nearest-neighbor hopping, i.e., using $\sigma=B_{n}$ $\times \exp \left(-\epsilon_{3} / k T\right)$ and $\epsilon_{3}=C_{n} N_{D}^{1 / 3}$, where $B_{n}$ and $C_{n}$ are temperature independent constants, the resulting $N_{D}$ of (4-8) $\times 10^{21} \mathrm{~cm}^{-3}$ for all three samples is unreasonably high. This is similar as reported previously. ${ }^{1}$ A comparison of a variable range single phonon and a multiphonon fits for samples $\mathrm{S} 1$ and S3 are shown in Fig. 3 (corresponding data for S2 are nearly identical to $\mathrm{S} 1$ and therefore not shown). Assuming variable range hopping with $\sigma=B_{v} \exp \left[-\left(T_{0} / T\right)^{1 / 4}\right]$ and $T_{0}=C_{v} N_{D}^{-2 / 3}$, we get $N_{D}=5 \times 10^{15}$ and $1 \times 10^{15} \mathrm{~cm}^{-3}$ for S3 and S1 (S2) samples. These values are more reasonable, but fitting for sample $\mathrm{S} 3$ is not satisfying and according to hopping theory the variable range type should be observed only at low temperatures. However, conduction in low temperature (LT) grown nonstoichiometric MBE GaAs can be well described by variable range hopping at room temperature. On the other hand, it is less probable that the defect concentration in highly resistive GaN and LT GaAs can be compared. Multiphonon hopping was recently considered as more reasonable to explain the conduction in highly resistive GaN. ${ }^{1}$ For this type of hopping a combination of both, nearest-neighbor and variable range single phonon hoppings, should occur. Fitting of our data to the equation $\sigma$ $=B_{\mathrm{mp}} \exp \left[-\left(T_{0} / T\right)^{1 / 4}\right] \exp \left(\epsilon_{3} / k T\right)$, shown in Fig. 3, is much better, mainly for sample S3. However, resulting $T_{0}$ 
hopping in GaN needs to be studied in more detail.

In conclusion, we performed temperature dependent conductivity and Hall effect measurements on highly resistive GaN layers grown by MBE. The main results of our investigation can be summarized as follows:

(i) Conductivity and Hall carrier concentration show thermally activated dependences and for all three samples investigated the same activation energy of $0.23 \mathrm{eV}$ is evaluated from the temperature dependent Hall concentration. Similar defect levels have been found recently in highly resistive GaN layers prepared by ion implantation.

(ii) The Hall mobility of samples with resistivity about $3 \times 10^{3} \Omega \mathrm{cm}$ shows that a scattering on charged dislocations dominates $\left(\mu_{H} \sim T^{x}, x=0.9\right.$ and 0.5$)$ in contradiction to lower resistive sample $(\rho=32 \Omega \mathrm{cm})$ for which phonon scattering dominates $(x=-1.4)$.

(iii) From the hopping analysis it can not be determined whether conduction in highly resistive $\mathrm{GaN}$ is nearestneighbor, variable range single phonon, or multiphonon hopping.

${ }^{1}$ D. C. Look, D. C. Reynolds, W. Kim, O. Aktas, A. Botcharev, A. Salvador, and H. Morkoç, J. Appl. Phys. 80, 2960 (1996).

${ }^{2}$ J. B. Webb, H. Tang, S. Rolfe, and J. A. Bardwell, Appl. Phys. Lett. 75, 953 (1999).

${ }^{3}$ N. I. Kuznetsov, A. E. Nikolaev, A. S. Zubrilov, Yu. V. Melnik, and V. A. Dimitriev, Appl. Phys. Lett. 75, 3138 (1999).

${ }^{4}$ Ch. Wang and R. F. Davis, Appl. Phys. Lett. 63, 990 (1993).

${ }^{5}$ R. J. Molnar, T. Lei, and T. D. Moustakas, Appl. Phys. Lett. 62, 72 (1993).

${ }^{6}$ H. M. Ng, D. Doppalapudi, T. D. Moustakas, N. G. Weimann, and L. F. Eastman, Appl. Phys. Lett. 73, 821 (1993).

${ }^{7}$ D. C. Look and J. R. Sizelove, Phys. Rev. Lett. 82, 1237 (1999).

${ }^{8}$ J. M. Van Hove, G. J. Cosimini, E. Nelson, A. M. Wowchak, and P. P. Chow, J. Cryst. Growth 150, 908 (1995).

${ }^{9} \mathrm{M}$. Grimm (private communication).

${ }^{10}$ X. A. Cao, S. J. Pearton, G. T. Dang, A. P. Zhang, F. Ren, R. G. Wilson, and J. M. Van Hove, J. Appl. Phys. 87, 1091 (2000).

${ }^{11}$ B. Pödör, Phys. Status Solidi 16, K167 (1966). 